Archives

$37 \mid 2006$

Quoi de neuf en Histoire Ancienne ? Le diable au Moyen Âge

\title{
Du laboratoire scolastique aux formes modernes de la démonologie
}

Sophie Houdard

\section{(2) OpenEdition \\ Journals}

Édition électronique

URL : http://journals.openedition.org/ccrh/3121

DOI : $10.4000 /$ ccrh.3121

ISSN : 1760-7906

Éditeur

Centre de recherches historiques - EHESS

Édition imprimée

Date de publication : 1 juin 2006

Pagination : 181-188

ISSN : 0990-9141

Référence électronique

Sophie Houdard, «Du laboratoire scolastique aux formes modernes de la démonologie », Les Cahiers du Centre de Recherches Historiques [En ligne], 37 | 2006, mis en ligne le 22 juin 2011, consulté le 21 avril 2019. URL : http://journals.openedition.org/ccrh/3121; DOI : 10.4000/ccrh.3121

Ce document a été généré automatiquement le 21 avril 2019

Article L.111-1 du Code de la propriété intellectuelle. 


\title{
Du laboratoire scolastique aux formes modernes de la démonologie
}

\author{
Sophie Houdard
}

1 Qui entreprendrait de lire Satan hérétique du point de vue de l'époque moderne en prenant tous les risques de mécompréhension et d'ignorance y est d'une certaine manière invité par Alain Boureau qui prévoit que cette « histoire intellectuelle » offrira des outils à ceux qui «se sont efforcés de rétablir des continuités entre le christianisme médiéval et les formes diverses de réforme et de Contre-Réforme ${ }^{1}$. Ce sont donc quelques continuités que je voudrais envisager, en m'appuyant sur ce postulat de départ dont je voudrais suivre rapidement l'évolution aux XVIeet XVII siècles :

Le souci des démons serait né de la conjonction, de l'actualisation et de l'interaction de deux thème anciens, celui du pacte conclu avec le diable et celui de la possession. (p. 13)

\section{De la latence à la pulsion démoniaque : quand le diable sort du laboratoire}

Comme l'indique le titre courant du livre, Alain Boureau entreprend de mettre au jour la "naissance de la démonologie ", pour en saisir une origine à la fois précoce, brutale et spéculative. Il s'agit d'en finir avec la légende forgée au XIX siècle, mais persistante, d'un Moyen Âge chrétien globalement démoniaque et obscurantiste, qui interdit de prendre le diable au sérieux; au contraire, l'ouvrage choisit de l'observer dans son laboratoire scolastique, quand la construction de l'hérésie à Rome, les analyses logico-linguistiques universitaires et les théories dissidentes d'un Pierre de Jean Olivi par exemple, ont servi à analyser et à inventer une première forme à la démonologie. Cette "préhistoire » commence à la fin du XIII siècle ; courageusement, l'auteur montre qu'elle émerge non de la philosophie scolastique, mais qu'elle constitue un «tournant» démoniaque à l'intérieur des recherches spéculatives scolastiques. C'est bien sûr cette prise au sérieux 
d'une préhistoire philosophico-théologique qui fournit la thèse forte et nouvelle de ce livre.

Dans la préface aux Sciences du diable, publié en 1992 aux éditions du Cerf, Alain Boureau faisait déjà l'aveu d'une relation personnelle et compliquée avec le diable. Partant de cette croix pour l'historien à nouer ensemble le collectif et le singulier, il écrivait :

En termes simples, un médiéviste - en tous cas le médiéviste que je suis - n'arrive pas à saisir comment tel ou tel héritier de saint Thomas, de Guillaume d'Ockham ou de Grégoire de Rimini a pu loger dans le moule de l'admirable savoir médiéval des croyances aussi frustes et surtout les développer en des systèmes explicites et argumentés [...] Comment imaginer, depuis un tel sommet, une chute aussi brusque? $»^{2}$

Pour mieux comprendre le lien dérangeant pour l'historien médiéval entre les «croyances collectives frustes» et la singularité des nobles systèmes philosophiques, Alain Boureau insistait sur la nécessité de comprendre historiquement l'émergence de la démonologie, "mais à condition d'en maintenir l'étrangeté par rapport à la pensée scolastique ", et de saisir un mode d'« apparition brusque, dans la discontinuité la plus étrange ». La fin de la préface annonçait donc déjà le chantier du Satan hérétique en proposant de voir dans la démonologie moderne la mise en œuvre d'un « monodémonisme chrétien » universalisant, élaboré plus tôt dans la période de « latence des conflits médiévaux» (sur la personne humaine, par exemple, ou la psychologie aristotélicienne au XIII siècle). La contribution au colloque sur le sabbat avait commencé d'analyser les racines scolastiques de l'anthropologie qui sous-tend la construction sabbatique ${ }^{3}$; la même année paraissait "Satan et le dormeur " ${ }^{4}$, article-clé dans cette histoire intellectuelle du diable, puisque la connection entre l'anthropologie de la personne et la "capacité » diabolique y était élaborée grâce au cas-limite du somnambule : le diable que construit la scolastique est donc au cœur des définitions du sujet, de sa responsabilité, de son aliénation et c'est cette construction anthropologique qui allait être le punctum de cette histoire. Enfin, en 1999, Théologie, science et censure au XIII - siècle était consacré à un certain nombre de ces conflits philosophiques, institutionnels et disciplinaires.

5 Satan hérétique, comme l'annonçait la préface de 1992, met donc enfin au jour le "tournant démoniaque», comme moyen de lutte et de résolution de ces conflits philosophiques au sein de l'édifice noble de la scolastique que le diable, devenu maitre du puzzle, amènera bientôt et comme toujours à faire chuter : dès le $\mathrm{XV}^{\mathrm{e}}$ siècle, le diable, on le sait, sort de sa période de latence, l'époque moderne étant à la fois résolution et passage à la pulsion violente, la witch-craze.

6 La première question que se posera un moderniste porte sur ce schéma d'une période de "latence», qui, comme tout freudien le sait, est une période d'inactivité et de refoulement. "latence » et "retard à l'allumage », selon les expressions retenues par Alain Boureau, disent assez la difficulté à expliquer l'écart entre la genèse de l'obsession démoniaque et la witch craze qui s'empare de l'Europe, dans une véritable folie persécutrice, à partir du XVe siècle. On filera donc ici la métaphore freudienne envisagée dès 1992 par Alain Boureau : pourquoi ces manifestations de "poussées » démoniaques qui renoncent à une organisation, en l'occurrence judiciaire, disciplinaire, de la démonologie? Les lourds conflits (dont la condamnation de thèses hétérodoxes publiées 
par l'évêque Etienne Tempier en 1277 (p. 12), la crise qui secoue l'ordre franciscain, etc.) trouvent-ils dans le diable de quoi bloquer des débats que l'autorité, d'une manière ou d'une autre, réprime ou contient? On sait que c'est après les conciles de Bâle et de Constance, au XVe siècle, que l'allumage prend et que la pulsion démoniaque démarre : ne faudrait-il pas envisager ces conciles comme de véritables forums publics de la démonologie, dont sortiront les grands manuels (le Formicarius de Nider, puis Sprenger) qui permettront la publication, à large échelle, des hypothèses démoniaques jusque-là confinées par l'autorité dans le champ clos du débat et de la censure scolastique? Les énonciations savantes trouveraient à se "réaliser » dès lors qu'elles sont publiquement développées et diffusées et qu'elles donnent lieu à des pratiques, des techniques qui visent aussi à étouffer le discours antidémonologique qui trouvera des voies plus dissimulées pour se publier à son tour.

\section{L'innocuité de la formation intellectuelle}

7 Le «souci des démons » commence avec l'anxiété du pape Jean XXII, sa volonté de savoir qu'on pourraitdire daimonocentrée. Comme le montre l'exemple de l'évêque de Cahors, Hugues Géraud, accusé en 1317 d'avoir voulu attenter à la vie du pape à l'aide d'un maleficium, on peut rapporter cette obsession du pontife à la crainte d'une magie dont l'efficacité (sous la forme du crime politique) est redoutée. Il s'agit d'une terreur ancienne dont les codes de Théodose et de Justinien font déjà état et qui élaborent la notion vouée à un long avenir de crimen majestatis.

8 La concomitance entre volonté de savoir, violence institutionnelle et idéologique est ancienne, mais elle prend un nouvel essor sous le pontificat de Jean XXII, par la consultation, voulue par le pape lui-même en 1320, chargée de fonder la sorcellerie et la magie en hérésie. Enrico del Caretto construit, comme le montrent les documents étudiés par Alain Boureau, une efficacité du pacte diabolique sur le modèle du pacte sacramentel, dotant le diable d'une puissance surnaturelle de subversion religieuse, sociale et politique, puisque c'est toute la société qui est fondée sur le pacte et le serment. La démonologie fabriquée par la consultation, puis qualifiée par la bulle Super illius specula (1326-1327), n'est pas une extension de l'hérésie (p. 75), puisqu'elle sort du domaine strict de l'erreur pour être objectivée et devenir un fait qui dépend d'un pacte fort avec le diable (comme le montre l'invention du verbe actif "hérétiquer»). La crainte de l'autorité et l'obsession du complot conduisent à la factualité objective qui autorisent la libération des démons; celle-ci peut alors (potentialité toute intellectuelle, mais potentialité tout de même) avoir lieu dans le monde où vivent les hommes grâce à l'élaboration doctrinale d'une "démonologie scolastique». Dans un mouvement d'introversion, la théorie du complot conduit à penser que l'invasion des diables qui se fait dans l'homme (par possession, incorporation) doit être « réactualisée » (p. 13) pour penser des sujets capables de s'ouvrir aux diables, dans la béance de sujets dont la volonté est fragilisée, et potentiellement aliénée. Le pacte, la possession, l'assujettissement sont les pièces maîtresses des enquêtes que mèneront plus tard l'inquisition et les tribunaux laïques.

9 Cette première démonologie est construite par Alain Boureau comme la résultante d'un faisceau de conflits entre les théologiens et les artiens, qui oppose la théologie et le naturalisme, entre les Spirituels et la papauté, entre des philosophies alternatives : elle en est à la fois le produit et le terme, sans qu'on sache véritablement d'ailleurs ce qui produit 
la naissance de la démonologie. Les conflits, dont la virulence démoniaque, on l'a vu, est latente, construisent dans l'ouvrage des séries parallèles, parfois convergentes, souvent concomitantes, selon une histoire complexe de la censure qui évalue parfois des défauts de raisonnement, parfois des auteurs, selon un régime stratifié de l'infidelitas et pour des jugements de science ou de foi. Si le diable de la première démonologie permet, selon Alain Boureau, d'accéder à une histoire de la liberté de discussion, il est aussi le moyen d'observer son contrôle, ne serait-ce que par la mise en place de lieux différenciés et conflictuels du magistère.

Satan hérétique s'en tient volontairement à l'étude d'une formation intellectuelle. Dans l'Épilogue, l'auteur écrit que cette genèse a finalement coûté cher, soit le prix exorbitant a posteriori de cette science de l'homme, "véritable innovation scolastique » (p. 262) qui a fait naître un diable pour penser une anthropologie de la personne et d'un sujet complexe, et donc déjà moderne. L'idée avait d'ailleurs été formulée dès le colloque sur le sabbat:

[la chasse aux sorcières aurait] inscrit dans l'antique langage de la possession satanique le scénario neuf de la construction du sujet moderne, dans le face à face interminable de l'individu et de la communauté ${ }^{5}$.

La deuxième question s'articulera alors à la première: pour les besoins de la démonstration, il fallait isoler le diable dans son laboratoire philosophico-théologique, créer un vide, une zone stérile. D'où aussi l'hypothèse d'une innocuité de cette genèse intellectuelle, comme si le diable avait muté, plus tard, en sortant du laboratoire et s'offrait alors à une autre histoire, celle du face à face répressif des pratiques judiciaires et des enquêtes des tribunaux modernes.

Dans leur introduction au numéro de Médiévales consacré au "Diable en procès ${ }^{6}$, Martine Ostorero et Étienne Anheim écrivent que les différences et les analogies entre le Moyen Âge et la première modernité « incitent à faire une histoire politique du diable » et je leur donnerais volontiers raison. La construction d'une première culture démoniaque est certes spéculative, mais elle s'élabore au moment où s'installent durablement des conflits de légalité, de responsabilité des idées et, ce qui va d'un même pas, le contrôle de leur publication. La libération des démons et leur invasion dans le sujet mettent déjà en place la matrice de l'Inquisition qui produit l'obéissance nécessaire à la construction de l'absolutisme moderne, en donnant le droit d'enquêter sur la fama.Le vide nécessaire à la démarche ne dit-il pas en creux une nouvelle fois, l'impossibilité à nouer le «singulier " sublime au collectif des "croyances frustes»? Une histoire intellectuelle, limitée à l'étude de discours ou d'énoncés ne risque-t-elle, si l'on n'élabore pas une histoire de leurs effets et donc de leur action, de renouveler, en voulant les éviter, les impasses de l'histoire des mentalités ? La distinction que feront d'ailleurs bon nombre de suspects à l'époque moderne entre magie savante et magie populaire ne reconduit-elle pas un séparatisme plus protecteur que réel?

\section{Le fait hérétique à l'époque moderne}

Le tournant du XVI ${ }^{e}$ au XVII siècle apporte à la fois la dernière formation théorique de la démonologie et ses premières impasses. Jean Bodin écrit en 1580 la Démonomanie des sorciers, soit le dernier traité, au sens générique, de démonologie ; Pierre de Bérulle écrit 
le dernier traité de la possession en français, Le Traicté des énergumènes en 1599. Après ces traités théologico-politico-juridiques naitront des sous-genres de la démonologie (les Histoires vraies, par exemple) qui participeront, souvent malgré leurs auteurs, à la démolition du modèle théorique et doctrinal.

Bodin est le dernier, en tous cas en France, à proposer des définitions du fait et donc à poser son existence. En juriste, il énumère le «fait permanent ", le «fait évident ", le « fait transitoire » qui articulent, selon une hiérarchie de la notoriété, un système de la preuve. Pour Bodin, le fait de sorcellerie exige une procédure exceptionnelle qui autorise tous les pièges, tous les moyens déceptifs pour arriver au fait, boucler la preuve et forcer le secret de la secte. Mais à cette quête obstinée du fait qui prouve, Bodin substitue, dans le même chapitre, la mention du pacte seul, qu'il appelle « l'obligation au diable », et qui mérite selon lui indépendamment de tout délit, la peine de mort. Le complot subversif, sous le chef d'accusation du crime de lèse-majesté divine et humaine, situé dans l'intention, suffit à condamner. À la preuve objective du fait notoire, Bodin substitue donc l'intention mauvaise, le projet pur, quasiment pervers, de l'erreur volontaire, comme subversion politique qu'il est nécessaire de faire avouer par tous les moyens.

Or, comme l'a montré Alfred Soman ${ }^{7}$, c'est le fait comme preuve, qui finit paradoxalement par décriminaliser la sorcellerie. Soman estime en effet que bien avant de douter de la réalité de la sorcellerie elle-même, c'est la méfiance dans l'administration de la preuve qui l'emporte chez les juges. Le fait qui a permis de construire la sorcellerie en un délit d'hérésie objectif, en un seul délit criminel capable de réduire la poussière des actions transitoires et secrètes, est au début du XVII siècle ce qui déconstruit le délit lui-même : le fait, qui a permis de s'assurer de l'opposition binaire du vrai et du faux, défait, à proportion même du rôle qu'on lui a fait jouer, l'édifice juridique de la démonologie.

À l'âge des confessions, au moment où s'élabore sous la plume du même Bodin la doctrine de la souveraineté, l'histoire du fait (passé dans les mains de la justice laïque) montre que, peu à peu, il ne suffit plus à établir une objectivation du vrai. Le fait, comme preuve ne marche pas et il rejoint dangereusement la poussière des notes d'hérésie qui ont envahi de leur côté la doctrine des canonistes, le feuilleté infini de ce qu'on appelle désormais l'orthodoxie, seul moyen, mais aporétique, de surmonter les impasses de l'hérésiologie traditionnelle. Le fait hérétique, comme le montre Alain Boureau, inventé comme moyen de lutte contre le "fait faible de l'intention" au XIII siècle, (p. 44), devient inefficace à l'âge moderne et vient se loger dans l'intention, le for interne, la conscience toujours dissimulée des sujets que canonistes, casuistes et juristes laïques traquent par tous les moyens.

17 Restent le pacte "fort ", seulement et terriblement intentionnel, et l'obsession conjointe du complot et de la faction dont le diable n'est plus qu'un comparse nécessaire encore à un absolutisme moderne construit sur un modèle théologico-politique.

Mais que devient toute cette affaire dans la théologie ? Les débats soulevés dans l'ouvrage entre le magistère et les Spirituels, entre la théologie et des doctrines franciscaines ou issues de la mystique rhénane (si l'on pense à Eckhart condamné par la bulle In Agro dominico de 1329, aux débats sur la vision béatifique, etc.) me paraissent avoir des prolongements quand le thomisme domine à l'âge moderne. Les débats sur la doctrine de l'acte dès la fin $\mathrm{du} \mathrm{XVI}_{\mathrm{I}}$ siècle (qui posent les premières coordonnées de la querelle du quiétisme au XVII siècle) ne déplacent-ils pas, sur la longue durée, la question du fait hérétique? C'est la démarche du sujet, démarche d'ordre quasi psychologique devant les 
objets de la foi, qui détermine désormais l'acceptable et le scandale. La mise en place d'une anthropologie de la personne d'origine scolastique trouverait ici une suite, un prolongement mystique ou spirituel. Satan hérétique met en série un faisceau de questions philosophiques et théologiques qui me paraît se scinder au XviI siècle, encore que le traitement judiciaire et les questions posées par les spirituels se fassent sur le même fond de psychologisation ou d'involution au profit d'enquêtes qui portent sur le sujet (sujet dans l'État, sujet dans le droit, sujet dans la foi). Il reste qu'au début de l'âge moderne, on assiste à un éclatement de cette mise en série : le droit et le politique se saisissent du diable - comme masque - pour régler des affaires politiques. Mais le diable, un autre diable ( ?) fait retour au XVII e siècle dans les grandes affaires de possession, où il devient un sérieux fauteur de trouble dans la forteresse thomiste, en se faisant l'allié de spiritualités alternatives : le diable devient ou redevient le moyen de penser des modèles alternatifs de la personne, du sujet; ce faisant, il retisse ensemble anthropologie, ecclésiologie et théologie.

19 Je donnerai quelques exemples. Les volumineuses histoires de l'hérésie du début du XVII siècle construisent les origines médiévales des demi-hérésies modernes. On y retrouve pêle-mêle le libre esprit, les béguines, les bégards, les Fraticelles, les Alumbrados, les magiciens, etc. Dans ce pêle-mêle, on observe, outre la permanence du complot et de la secte, l'insistance sur une doctrine de l'acte qui oppose intellectualisme et volontarisme. Bien des analogies existent donc entre les débats (sur la personne, la relation, la volonté) éclairées dans la genèse de la démonologie et ceux que soulèvent les spirituels à l'époque moderne: le primat de la volonté sur la raison, de l'affectus sur la connaissance rationnelle, de la relation contractuelle dans la vie mystique, l'importance du modèle dionysien et d'une tradition d'origine rhénane, les moyens courts de voir Dieu, etc.

Les spirituels soumis aux corrections, aux explications, voire aux interdictions, élaborent une doctrine de l'acte qui s'appuie sur une tradition spirituelle médiévale, pour placer la négation de la connaissance non sur les objets de la foi, mais sur les modalités du sujet qui opère cet acte de connaissance. Jusqu'à la bulle Coelestis Pastor de 1687 qui signe la victoire de l'antiquiétisme et de l'antimystique, la discussion sur les modalités de l'acte oppose thomistes et mystiques et deux traditions théologiques (une tradition officielle et une tradition "nouvelle», mystique). La présence d'un Pierre de Bérulle dans l'affaire de la possession de Marthe Brossier en 1599, celle du jésuite Jean-Joseph Surin à Loudun, la place de la doctrine de Canfield dans la possession de Louviers me paraissent faire de la possession un laboratoire in vivo de la personne. Cette possession, comme modèle mystique me paraît prolonger, jusqu'en plein XVII siècle, l'étude sismologique élaborée dans le Satan hérétique attentif aux fêlures de la « plaque » de la personne, dès lors qu'elle est soumise aux secousses de modèles alternatifs qui déverrouillent un "socle solide au monisme fondamental ».

D'où une troisième et dernière question: les doctrines de l'acte pourraient-elles constituer le prolongement moderne, mystique de cette anthropologie de la personne dont le diable a été le laboratoire et dont la possession devient le lieu expérimental au XVII siècle ? comme si le diable du XVII siècle, parent lointain du Satan hérétique, issu du tournant brutal et nouveau de la construction scolastique, nous obligeait à penser une histoire longue, faite de séries parallèles (histoire juridico-politique, histoire spirituelle) où les continuités, malgré les apparences, sont plus fortes que les ruptures. 


\section{NOTES}

1. Alain Boureau, Satan hérétique, p. 19. Désormais, les références à cet ouvrage seront indiquées entre parenthèses dans le corps du texte.

2. Sophie Houdard, Les Sciences du diable Quatre discours sur la sorcellerie, Paris, Le Cerf, 1992, préface, p. 10.

3. Alain Boureau, «Le sabbat et la question scolastique de la personne », Le sabbat des sorciers $X V^{e_{-}}$ XVIII' siècles, Grenoble, Jérôme Millon, 1993.

4. Alain Boureau, "Satan et le dormeur une construction de l'inconscient au Moyen Âge ", Chimères, 14, 1991-1992, p. 41-61.

5. Alain Boureau, Le sabbat, art. cit., supra note 2, p. 46.

6. Martine Ostorero, Étienne Anheim, « Le diable en procès ", Médiévales, 44 (2003), p. 5-16.

7. Alfred Soman, Sorcellerie et justice criminelle : le Parlement de Paris (16 $-18^{e}$ siècles), Aldershot, Variorum (Collected studies series, 368), 1992.

\section{AUTEUR}

\section{SOPHIE HOUDARD}

Université Paris-III 